WIS-93/110/Nov-PH

TAUP-2117-93

hep-ph/9311250

\title{
Quark Solitons from effective action of QCD
}

\author{
YITZHAK FRISHMAN $^{\star}$ AND AMIHAY HANANY ${ }^{\dagger}$ \\ Department of Particle Physics \\ Weizmann Institute of Science \\ 76100 Rehovot Israel \\ MAREK KARLINER ${ }^{\ddagger}$ \\ School of Physics and Astronomy \\ Beverly and Raymond Sackler \\ Faculty of Exact Sciences \\ Ramat Aviv Tel-Aviv, 69987, Israel
}

\begin{abstract}
We derive an effective low energy action for QCD in 4 dimensions. The low energy dynamics is described by chiral fields transforming non-trivially under both color and flavor. We use the method of anomaly integration from the QCD action. The solitons of the theory have the quantum numbers of quarks. They are expected to be the constituent quarks of hadrons. In two dimensions our result is exact, namely the bosonic gauged action of WZW.
\end{abstract}

\footnotetext{
^ e-mail: fnfrishm@weizmann.bitnet

$\dagger$ e-mail: ftami@weizmann.bitnet

† e-mail: marek@vm.tau.ac.il
} 


\section{Effective action from anomaly integration}

The connection between the phenomenologically successful non-relativistic constituent-quark model (NRQM), and QCD's fundamental degrees of freedom continues to be one of the open and puzzling questions in particle physics. The constituent quarks which are the basic building blocks of the NRQM have the same quantum numbers as the much lighter and highly relativistic QCD current-quark fields. In addition, recent data from polarized deep inelastic lepton-nucleon scattering $^{[1-3]}$ and the ensuing conclusions about spin structure of the nucleon ${ }^{[4]}$ indicate that the constituent quarks are composite objects with internal structure. The emergence of constituent quarks as low-energy effective excitations of QCD is clearly due to a nonperturbative mechanism. Finding a theoretical description of this mechanism is an important and interesting challenge.

Some steps in this direction have already been made. A model combining some of the features of the chiral quark ${ }^{[5]}$ and skyrmion ${ }^{[6-8]}$ approaches has been constructed by Kaplan ${ }^{[9]}$. In this model it is postulated that at distances smaller than the confinement scale but large enough to allow for nonperturbative phenomena the effective dynamics of QCD is described by chiral dynamics of a bosonic field which takes values in $U\left(N_{c} \times N_{f}\right)$. This effective theory admits classical soliton solutions. Assuming that they are stable and may be quantized semiclassically, one then finds that these solitons are extended objects with spin $1 / 2$, and that they belong to the fundamental representation of color and flavor. Their mass is of

order $\Lambda_{Q C D}$ and radius of order $1 / \Lambda_{Q C D} .^{[10]}$ It is very tempting to identify them as the constituent quarks. Thus the constituent quarks in this model are "skyrmions" in color space.

This is a very attractive idea, but some crucial elements of the puzzle are still missing, most importantly a derivation of the effective dynamics from QCD. However, recent work on $\mathrm{QCD}$ in $1+1$ dimensions $\left(\mathrm{QCD}_{2}\right)$ provides evidence in support of this picture. ${ }^{[1]]}$ Exact nonabelian bosonization allows the Lagrangian of $\mathrm{QCD}_{2}$ to be re-expressed in terms of a chiral field $U(x) \in U\left(N_{c} \times N_{f}\right)$ and of the 
usual gauge field. This bosonized Lagrangian has topologically nontrivial static solutions that have the quantum numbers of baryons and mesons, constructed out of constituent quark and anti-quark solitons.

When $N_{c}$ solitons of this type are combined, a static, finite-energy, color singlet solution is formed, corresponding to a baryon. Similarly, static meson solutions are formed out of a soliton and an anti-soliton of different flavors. The stability of the mesons against annihilation is ensured by flavor conservation. These results can be viewed as a derivation of the constituent quark model in $\mathrm{QCD}_{2}$. Thus the idea of constituent quarks as solitons of a Lagrangian with colored chiral fields becomes exact in $D=1+1$.

In this paper we will provide a constructive scheme for deriving from QCD the relevant effective Lagrangian. In two dimensions the procedure will yield the exact bosonic action of $\mathrm{QCD}_{2}$, namely the gauged WZW Lagrangian. In four dimensions the resulting action turns out to be a chiral Lagrangian whose leading terms are very similar to the action conjectured by Kaplan.

The construction of the effective chiral action for bosonic variables is based on integration of the anomaly equation. ${ }^{[12-14]}$ The idea is to construct an action which will reproduce the anomaly under chiral transformations. One begins with the anomaly equation as the starting point and integrates it with respect to the chiral rotation parameter. The resulting action typically contains additional terms which are anomaly-free. These terms are analogous to an integration constant in an ordinary indefinite integral. Therefore their coefficients are arbitrary. There are also additional terms in the form of Chern-Simons action which are non-local.

In order to resolve this freedom, one may adopt a strategy analogous to computing a definite integral, in this case rotating the action by a finite chiral rotation. This rotated action is vector invariant by choice of scheme. When the change in external fields is compensated by a change in the chiral phase, the rotated action is also axial invariant. Next, taking the difference of the rotated and unrotated action, one gets the effective chiral action for bosonic fields. This action includes 
a term which is non-local, namely the Wess-Zumino term, which locally is a total derivative.

We will see that in 2 dimensions this procedure generates the gauged WZW action, while in 4 dimensions it generates a new type of low-energy effective action for chiral fields transforming non-trivially under both color and flavor. We will then demonstrate that baryons and constituent quarks are the solitonic excitations of such an action.

In order to carry out this program, we need to deal with several preliminary issues. First, we have to define the scheme in which the anomaly equation will be discussed. Having chosen the scheme, we also need to choose a suitable regularization prescription. In a given scheme the choice of the regularization cannot influence the results of an exact calculation. As we shall see, however, in 4 dimensions the effective action contains an infinite number of terms, corresponding to expansion in terms of powers of external momenta divided by the cutoff. If we truncate the expansion at a finite number of terms and take a finite cutoff, the result will be regularization dependent. In 2 dimensions, however, the limit of infinite cutoff reproduces the exact bosonized action of WZW.

Given an anomaly equation one can redefine the axial current and add a counter term which is an axial variation of a local action. ${ }^{[15]}$ This creates an ambiguity in the definition of the quantum axial current. So we need some principles which remove this ambiguity. The first one is our choice of the vector scheme, we want to remain with a vector-conserving theory. The second one is to keep the right relation between the axial and the vector currents. In two dimensions the axial current is the dual of the vector current $J_{5}^{\mu}=-\epsilon^{\mu \nu} J_{\nu}$. In 4 dimensions no such simple relation exist. We choose the heat kernel regularization. ${ }^{[16]}$ In 2 dimensions this procedure is well defined and the end result is a complete bosonization for gauged fermions. Unfortunately in 4 dimensions this cannot be done and the kinetic term is proportional to the cutoff squared. We therefore keep the UV cutoff finite and look for its physical meaning. This will be described later. The price we pay is 
that we remain with regularization dependent terms and a low energy expansion. The value of the UV cutoff will determine the range of energies for which this approximation is valid. What is remarkable is that these calculations reproduce very accurately the numbers calculated for chiral Lagrangians in the case of flavor

symmetry. ${ }^{[17]}$ It is still mysterious why the heat-kernel regularization fits the results of experiment ${ }^{[13,18]}$

The outline of the paper is as follows. In sec. 2 we introduce the notation and set up the general formalism. In sec. 3 we apply the formalism to derive the gauged WZW action in 2 dimensions. In sec. 4 we derive the low energy effective action for the chiral field in 4 dimensions. In section 5 we discuss various schemes of choosing the group in which the chiral field is embedded, new classical solutions in the "U-scheme", conserved currents including the baryon number current, and finally the Polyakov-Wiegmann formula extended to 4 dimensions. Its low energy approximation, up to dimension-4 operators, is given explicitly.

\section{General formulation}

In this section we set the notation and describe the general procedure. Those features which are special to 2 or 4 dimensions will be discussed in the relevant sections.

The fermionic part of the QCD Lagrangian with external sources coupled to vector and axial currents, scalar and pseudoscalar densities labeled $V_{\mu}, A_{\mu}, S, P$ respectively, has the form

$$
S_{F}=\bar{\psi}\left(i \not \partial-V-\not A \gamma_{5}-S-i \gamma_{5} P\right) \psi
$$

Where $V_{\mu} \equiv V_{\mu}^{a} T^{a}, A_{\mu} \equiv A_{\mu}^{a} T^{a}, S \equiv S^{a} T^{a}, P \equiv P^{a} T^{a}, T_{a} \in G$ are the generators of some group $\mathrm{G}$, normalized through $\operatorname{Tr}\left(T^{a} T^{b}\right)=\frac{1}{2} \delta^{a b}$. In the generic case we think of $G$ as the group $U\left(N_{c} \times N_{f}\right)$, where $N_{c}$ and $N_{f}$ are the number of colors and flavors, respectively. Other groups can also be chosen, as will be discussed 
below. $V$ will eventually include the dynamical gauge field. For later convenience we introduce the chiral fields

$$
\begin{aligned}
& L_{\mu}=V_{\mu}-A_{\mu}, \\
& R_{\mu}=V_{\mu}+A_{\mu} .
\end{aligned}
$$

At this stage we do not include a mass term, as this breaks the chiral symmetry explicitly, on top of the breaking due to anomaly. The variation of $S$ and $P$, on the other hand, is compensated by a rotation of $\psi$ (see below).

Since (2.1) is quadratic in the fermion fields, the fermions can be integrated out explicitly, yielding the determinant

$$
Z[V, A, S, P]=\int[D \psi][D \bar{\psi}] e^{i S_{F}}
$$

At this point we have some freedom of choice. The axial current cannot be conserved. We need to choose a scheme of conservation. Here, since at the end $V$ will contain the dynamical field for gluons, we choose $Z$ to be invariant under vector gauge transformations,

$$
\left\{\begin{array}{l}
\delta V_{\mu}=D_{\mu} \omega=\partial_{\mu} \omega+i\left[V_{\mu}, \omega\right] \\
\delta A_{\mu}=i\left[A_{\mu}, \omega\right] \\
\delta S=i[S, \omega] \\
\delta P=i[P, \omega] \\
\delta L_{\mu}=\partial_{\mu} \omega+i\left[L_{\mu}, \omega\right] \\
\delta R_{\mu}=\partial_{\mu} \omega+i\left[R_{\mu}, \omega\right]
\end{array}\right.
$$


On the other hand, the variation of the determinant under the axial transformation

$$
\left\{\begin{array}{l}
\delta V_{\mu}=i\left[A_{\mu}, \lambda\right] \\
\delta A_{\mu}=D_{\mu} \lambda=\partial_{\mu} \lambda+i\left[V_{\mu}, \lambda\right] \\
\delta S=-\{P, \lambda\} \\
\delta P=\{S, \lambda\} \\
\delta L_{\mu}=-\partial_{\mu} \lambda-i\left[L_{\mu}, \lambda\right] \\
\delta R_{\mu}=\partial_{\mu} \lambda+i\left[R_{\mu}, \lambda\right]
\end{array}\right.
$$

is anomalous. This non-invariance of $Z$ under axial transformation can be written in compact form as

$$
-i \frac{\delta \log Z}{\delta \lambda^{a}}=-\mathcal{A}^{a}[V, A, S, P]
$$

where $\mathcal{A}$ is the anomaly. We integrate this equation using the chain rule

$$
\frac{\delta Z}{\delta \lambda}=-D_{\mu} \frac{\delta Z}{\delta A_{\mu}}-i\left[A_{\mu}, \frac{\delta Z}{\delta V_{\mu}}\right]+\left\{S, \frac{\delta Z}{\delta P}\right\}-\left\{P, \frac{\delta Z}{\delta S}\right\}
$$

to get an action $Z_{1}$ which obeys

$$
-i \frac{\delta \log Z_{1}}{\delta \lambda^{a}}=-\mathcal{A}^{a}[V, A, S, P] .
$$

$Z$ can be written as a product $Z_{1} Z_{2}$, where $Z_{1}$ is not chiral invariant and can be calculated exactly from the anomalies, and $Z_{2}$ is chiral invariant and cannot be calculated via this procedure. We now make a right rotation with a chiral field $U$,

$$
\left\{\begin{array}{l}
L_{\mu}^{U}=L_{\mu} \\
R_{\mu}^{U}=U^{-1} R_{\mu} U-U^{-1} i \partial_{\mu} U \\
(S+i P)^{U}=(S+i P) U \\
(S-i P)^{U}=U^{-1}(S-i P)
\end{array}\right.
$$

and denote $Z^{U}=Z\left[V^{U}, A^{U}, S^{U}, P^{U}\right]$. Later on we will show that the vector invariance implies that such a rotation can be taken without loss of generality. 
Note that $U$ can be chosen in any subgroup of $U\left(N_{c} \times N_{f}\right)$. This point will be also discussed later.

We arrive at the connection

$$
\begin{gathered}
S_{F}=S_{\mathrm{eff}}-i \log Z_{2} \\
S_{\mathrm{eff}}=-i \log Z_{1}[V, A, S, P]+i \log Z_{1}\left[V^{U}, A^{U}, S^{U}, P^{U}\right] .
\end{gathered}
$$

Now let us observe the following. First, $Z_{2}$ contains important physical information in it. It has, in principle, any power of the external sources, therefore it contains non-trivial information on general correlation functions. Second, $Z_{2}$ does not depend on $\mathrm{U}$. Therefore all the $U$ physics is in $S_{\text {eff }}$. We will argue later that $U$ describes the dynamics of quark solitons or baryon solitons, therefore $S_{\text {eff }}$ describes their complete dynamics. Third, note that in 2 dimensions, since we get exact bosonization, $Z_{2}$ becomes trivial. In the next sections we will treat $S_{\text {eff }}$ only. Note that $\log Z^{U}$ is invariant under the transformation

$$
\left\{\begin{array}{l}
\Phi \rightarrow \Phi^{\tilde{U}} \\
U \rightarrow \tilde{U}^{-1} U
\end{array}\right.
$$

where $\Phi$ represents any of the fields $L_{\mu}, R_{\mu}, S, P$ in (2.9). In fact $\Phi^{U}$ itself is invariant. Note however that applying the variation of $\Phi$ in $\log Z_{1}$ reproduces the anomaly. $S_{\text {eff }}$ is invariant under the vector transformation

$$
\left\{\begin{array}{l}
R_{\mu} \rightarrow \tilde{U}^{-1} R_{\mu} \tilde{U}-\tilde{J}_{\mu} \\
L_{\mu} \rightarrow \tilde{U}^{-1} L_{\mu} \tilde{U}-\tilde{J}_{\mu} \\
S \pm i P \rightarrow \tilde{U}^{-1}(S \pm i P) \tilde{U} \\
U \rightarrow \tilde{U}^{-1} U \tilde{U}
\end{array}\right.
$$

where $\tilde{J}_{\mu}=i \tilde{U}^{-1} \partial_{\mu} \tilde{U}$. 
We now turn to explain why the right rotation is sufficient in (2.9). The generalization of (2.9) to arbitrary left and right phases is

$$
\left\{\begin{array}{l}
L_{\mu}^{g}=g_{L}^{-1} L_{\mu} g_{L}-g_{L}^{-1} i \partial_{\mu} g_{L} \\
R_{\mu}^{g}=g_{R}^{-1} R_{\mu} g_{R}-g_{R}^{-1} i \partial_{\mu} g_{R} \\
(S+i P)^{g}=g_{L}^{-1}(S+i P) g_{R} \\
(S-i P)^{g}=g_{R}^{-1}(S-i P) g_{L}
\end{array}\right.
$$

where $g$ denotes $\left(g_{L}, g_{R}\right)$ together. The effective action with both left and right rotations is

$$
S_{\text {eff }}\left[V, A, S, P, g_{L}, g_{R}\right]=-i \log Z_{1}[V, A, S, P]+i \log Z_{1}\left[R^{g}, L^{g}, S^{g}, P^{g}\right]
$$

Vector invariance (2.13) implies

$$
S_{\text {eff }}\left[V, A, S, P, g_{L}, g_{R}\right]=S_{\text {eff }}\left[V, A, S, P, 1, g_{L}^{-1} g_{R}\right]
$$

and we can define $U=g_{L}^{-1} g_{R}$ to obtain (2.11).

\section{The procedure in 2 dimensions}

In this section we calculate the effective action in 2 dimensions. In this case the limit of UV cutoff going to infinity is well defined. We use $\operatorname{Tr}\left(\gamma_{5} \gamma_{\mu} \gamma_{\nu}\right)=2 \epsilon_{\mu \nu}$, $\epsilon_{01}=1, g_{00}=-g_{11}=1$. The axial variation of the action in 2 dimensions is given by

$$
-i \frac{\delta \log Z}{\delta \lambda^{a}}=\frac{1}{4 \pi}\left[\epsilon^{\mu \nu}\left(F_{\mu \nu}-2 i\left[A_{\mu}, A_{\nu}\right]\right)^{a}+2\left(D_{\mu} A^{\mu}\right)^{a}-2\{S, P\}^{a}\right]
$$

$F_{\mu \nu}$ is given by

$$
F_{\mu \nu}=\partial_{\mu} V_{\nu}-\partial_{\nu} V_{\mu}+i\left[V_{\mu}, V_{\nu}\right]+i\left[A_{\mu}, A_{\nu}\right]=\frac{1}{2}\left(L_{\mu \nu}+R_{\mu \nu}\right),
$$


and we have introduced the left and right field strengths

$$
\begin{aligned}
& L_{\mu \nu}=\partial_{\mu} L_{\nu}-\partial_{\nu} L_{\mu}+i\left[L_{\mu}, L_{\nu}\right] \\
& R_{\mu \nu}=\partial_{\mu} R_{\nu}-\partial_{\nu} R_{\mu}+i\left[R_{\mu}, R_{\nu}\right] .
\end{aligned}
$$

Also, $D_{\mu} A_{\nu}=\partial_{\mu} A_{\nu}+i\left[V_{\mu}, A_{\mu}\right]$. Note the term $D_{\mu} A^{\mu}$ in (3.1), which originates from a $g_{\mu \nu}$ part rather than $\epsilon_{\mu \nu}$ part. This term can be calculated by recognizing that for $A=0$ the anomaly is $-\frac{1}{2 \pi} \epsilon^{\mu \nu} F_{\mu \nu}$ and, for $A \neq 0, F$ is replaced by the field strength of the new vector field $V_{\mu}+\epsilon_{\mu \nu} A^{\nu}$.

Using the chain rule eq. (2.7) we have an action which has the variation (3.1)

$$
\begin{aligned}
-i \log Z_{1}=S_{C S}[R]-S_{C S}[L] & +\frac{1}{2 \pi} \int \epsilon^{\mu \nu} \operatorname{Tr}\left(V_{\mu} A_{\nu}\right)-\frac{1}{2 \pi} \int \operatorname{Tr} A_{\mu} A^{\mu} \\
& +\frac{1}{2 \pi} \int \operatorname{Tr}\left(S^{2}\right)
\end{aligned}
$$

Where $S_{C S}$ is the Chern-Simons action (an extension to $2+1$ dimensions)

$$
S_{C S}[A]=\frac{1}{4 \pi} \int \epsilon^{\mu \nu \sigma} \operatorname{Tr}\left(A_{\mu} \partial_{\nu} A_{\sigma}+\frac{2}{3} i A_{\mu} A_{\nu} A_{\sigma}\right)
$$

which has the variation

$$
\delta S_{C S}[R]=\frac{1}{4 \pi} \int \epsilon^{\mu \nu} \operatorname{Tr}\left(\left(\delta R_{\mu}\right) R_{\nu}\right)+\frac{1}{4 \pi} \int \epsilon^{\mu \nu \sigma} \operatorname{Tr}\left(R_{\mu \nu}\left(\delta R_{\sigma}\right)\right)
$$

and when using $\delta R_{\mu}=\partial_{\mu} \omega+i\left[R_{\mu}, \omega\right]$ the variation is

$$
\delta S_{C S}[R]=\frac{1}{4 \pi} \int \epsilon^{\mu \nu} \operatorname{Tr}\left(\left(\partial_{\mu} R_{\nu}\right) \omega\right)
$$

We use the Chern-Simons anomaly contribution

$$
S_{C S}\left[R^{U}\right]-S_{C S}[R]=-\frac{1}{4 \pi} \int \epsilon^{\mu \nu} \operatorname{Tr}\left(\bar{J}_{\mu} R_{\nu}\right)+\frac{i}{12 \pi} \int \epsilon^{\mu \nu \sigma} \operatorname{Tr}\left(J_{\mu} J_{\nu} J_{\sigma}\right)
$$


where $J_{\mu}=U^{-1} i \partial_{\mu} U$ and $\bar{J}_{\mu}=-U J_{\mu} U^{-1}$, to calculate the effective action (2.11),

$$
\begin{aligned}
S_{\mathrm{eff}} & =\frac{1}{8 \pi} \int \operatorname{Tr}\left(J_{\mu} J^{\mu}\right)-\frac{i}{12 \pi} \int \epsilon^{\mu \nu \sigma} \operatorname{Tr}\left(J_{\mu} J_{\nu} J_{\sigma}\right) \\
& +\frac{1}{4 \pi} \int\left(g^{\mu \nu}+\epsilon^{\mu \nu}\right) \operatorname{Tr}\left[\bar{J}_{\mu} R_{\nu}-L_{\mu} U^{-1} R_{\nu} U+L_{\mu} J_{\nu}+L_{\mu} R_{\nu}\right] \\
& -\frac{1}{8 \pi} \operatorname{Tr}\left\{\left[U^{-1}(S-i P)\right]^{2}+[(S+i P) U]^{2}+2\left(P^{2}-S^{2}\right)\right\}
\end{aligned}
$$

Note that it is $R_{-}$that couples to the right fermion current, and $L_{+}$to the left one. As can be seen the action (3.9) is the gauged WZW action of level 1 . $^{[19]}$ We learn that by anomaly integration, the effective action is an exact bosonization of Dirac fermions. Hints for this were given in ref. [20]. Note that when $P \rightarrow 0$ and $S \rightarrow M$, we get a $U^{2}+U^{-2}$ term, but not the expected $U+U^{-1}$ term which corresponds to a fermionic mass term. This is because we included only effects that come from the anomaly, through the Jacobian of the change of variables in fermion sector, but not the explicit breaking coming from the mass term.

\section{The procedure in 4 dimensions}

We now turn to 4 dimensions. We follow exactly the same procedure as in the 2 dimensional case but here we encounter some difficulties. While the result of eq. (3.9) is an exact bosonization of fermions coupled to vector and axial sources, its 4-dimensional analogue is an approximation, valid only at low energies. We use an expansion in powers of the cutoff, assuming the higher terms are suppressed by powers of the cutoff squared. The scale at which the approximation breaks down is determined by $\Lambda$, the ultraviolet cutoff in the regularization procedure.

Let us now go over to the derivation. The variation of the determinant under the axial transformation (2.5), including terms up to zeroth power of the cutoff, is 
given by ${ }^{[21,12]}$

$$
\begin{aligned}
-i \frac{\delta \log Z}{\delta \lambda^{a}} & =\frac{1}{4 \pi^{2}}\left\{\epsilon ^ { \mu \nu \lambda \sigma } \left[F_{\mu \nu} F_{\lambda \sigma}+\frac{1}{3} H_{\mu \nu} H_{\lambda \sigma}-\frac{4}{3}\left(A_{\mu} A_{\nu} F_{\lambda \sigma}+A_{\mu} F_{\nu \lambda} A_{\sigma}\right.\right.\right. \\
\left.+F_{\mu \nu} A_{\lambda} A_{\sigma}\right) & \left.+\frac{8}{3} A_{\mu} A_{\nu} A_{\lambda} A_{\sigma}\right]+16 \Lambda^{2} D_{\mu} A^{\mu}+\frac{2}{3}\left[D^{\mu} F_{\mu \nu}, A^{\nu}\right]+\frac{1}{3}\left[F_{\mu \nu}, D^{\mu} A^{\nu}\right] \\
& +\frac{1}{3}\left\{D^{\mu} A_{\mu}, A_{\nu} A^{\nu}\right\}+2 A_{\mu} D_{\nu} A^{\nu} A^{\mu}+\frac{1}{6}\left[\left[A_{\mu}, A_{\nu}\right], H^{\mu \nu}\right] \\
+ & \left.\frac{1}{3}\left(D_{\mu} A_{\nu} A^{\mu} A^{\nu}+A_{\mu} A_{\nu} D^{\nu} A^{\mu}\right)+D_{\mu} A_{\nu} A^{\nu} A^{\mu}+A_{\mu} A_{\nu} D^{\mu} A^{\nu}\right\}+\mathcal{O}\left(\Lambda^{-2}\right)
\end{aligned}
$$

where $H_{\mu \nu}=\frac{1}{2}\left(R_{\mu \nu}-L_{\mu \nu}\right)$. In (4.1) we have terms which are contracted with the metric rather than with the anti-symmetric tensor. Their coefficients are determined from a heat-kernel regularization procedure. ${ }^{[12,16]}$ We do not have here a relation like in 2 dimensions between vector and axial currents, and therefore we do not see simple relations among the various terms in (4.1). Using the chain rule (2.7), an action which has the above variation is given by

$$
\begin{aligned}
-i \log Z_{1} & =-\frac{2 \Lambda^{2}}{\pi^{2}} \int \operatorname{Tr}\left(A_{\mu} A^{\mu}\right)+S_{C S}^{5}[R]-S_{C S}^{5}[L] \\
& +\frac{i}{48 \pi^{2}} \int \epsilon^{\mu \nu \lambda \sigma} \operatorname{Tr}\left[i\left(R_{\mu \nu}+L_{\mu \nu}\right)\left\{L_{\lambda}, R_{\sigma}\right\}+\left(L_{\mu} L_{\nu}-\frac{1}{2} L_{\mu} R_{\nu}+R_{\mu} R_{\nu}\right) L_{\lambda} R_{\sigma}\right] \\
& -\frac{1}{12 \pi^{2}} \int \operatorname{Tr}\left\{-\frac{1}{4}\left(F_{\mu \nu}\right)^{2}+F^{\mu \nu}\left[A_{\mu}, A_{\nu}\right]+\frac{1}{2}\left(D_{\mu} A^{\mu}\right)^{2}-\left(A_{\mu} A^{\mu}\right)^{2}\right\}
\end{aligned}
$$

where $S_{C S}^{5}[R]$ is the Chern-Simons action in 5 dimensions (see for example the review in Ref. [22].)

$S_{C S}^{5}[R]=\frac{1}{24 \pi^{2}} \int \epsilon^{\mu \nu \lambda \sigma \rho} \operatorname{Tr}\left(R_{\mu} \partial_{\nu} R_{\lambda} \partial_{\sigma} R_{\rho}+\frac{3}{2} i R_{\mu} R_{\nu} R_{\lambda} \partial_{\sigma} R_{\rho}-\frac{3}{5} R_{\mu} R_{\nu} R_{\lambda} R_{\sigma} R_{\rho}\right)$

After rotation by $U$ we get the final result eq. (2.11), 


$$
\begin{aligned}
& S_{\text {eff }}=\frac{\Lambda^{2}}{2 \pi^{2}} \int \operatorname{Tr}\left[J_{\mu} J^{\mu}+2 \bar{J}_{\mu} R^{\mu}-2 L_{\mu} U^{-1} R^{\mu} U+2 L_{\mu} J^{\mu}+2 L_{\mu} R^{\mu}\right] \\
&-\frac{1}{240 \pi^{2}} \int \epsilon^{\mu \nu \lambda \sigma \rho} \operatorname{Tr}\left(J_{\mu} J_{\nu} J_{\lambda} J_{\sigma} J_{\rho}\right) \\
&+\frac{i}{48 \pi^{2}} \int \epsilon^{\mu \nu \lambda \sigma} \operatorname{Tr}\left\{i R_{\mu \nu}\left\{R_{\lambda}, \bar{J}_{\sigma}\right\}-R_{\mu} R_{\nu} R_{\lambda} \bar{J}_{\sigma}\right. \\
&\left.+\frac{3}{2} R_{\mu} \bar{J}_{\nu} R_{\lambda} \bar{J}_{\sigma}+\bar{J}_{\mu} \bar{J}_{\nu} \bar{J}_{\lambda} R_{\sigma}\right\} \\
&+\frac{i}{48 \pi^{2}} \int \epsilon^{\mu \nu \lambda \sigma} \operatorname{Tr}\left\{i\left(R_{\mu \nu}+L_{\mu \nu}\right)\left\{L_{\lambda}, R_{\sigma}\right\}+\left(L_{\mu} L_{\nu}-\frac{1}{2} L_{\mu} R_{\nu}+R_{\mu} R_{\nu}\right) L_{\lambda} R_{\sigma}\right\} \\
&-\frac{i}{48 \pi^{2}} \int \epsilon^{\mu \nu \lambda \sigma} \operatorname{Tr}\left\{i\left(U^{-1} R_{\mu \nu} U+L_{\mu \nu}\right)\left\{L_{\lambda}, R_{\sigma}^{U}\right\}+\left(L_{\mu} L_{\nu}-\frac{1}{2} L_{\mu} R_{\nu}^{U}+R_{\mu}^{U} R_{\nu}^{U}\right) L_{\lambda} R_{\sigma}^{U}\right\} \\
&+\frac{1}{192 \pi^{2}} \int \operatorname{Tr}^{U}\left[-2\left(D_{\mu} U^{-1} D^{\mu} U\right)^{2}+\left(D_{\mu} U^{-1} D_{\nu} U\right)^{2}+2\left(D_{\mu} D^{\mu} U^{-1}\right) D_{\nu} D^{\nu} U\right. \\
&\left.+4\left(R_{\mu \nu} D^{\mu} U^{-1} D^{\nu} U+L_{\mu \nu} D^{\mu} U D^{\nu} U^{-1}\right)+U^{-1} L_{\mu \nu} U R^{\mu \nu}-L_{\mu \nu} R^{\mu \nu}\right] \\
& \quad+16\left\{F^{\mu \nu}\left[A_{\mu}, A_{\nu}\right]+\frac{1}{2}\left(D_{\mu} A^{\mu}\right)^{2}-\left(A_{\mu} A^{\mu}\right)^{2}\right\}
\end{aligned}
$$

where $D_{\mu} U=\partial_{\mu} U+i R_{\mu} U-i U L_{\mu}, D_{\mu} U^{-1}=\partial_{\mu} U^{-1}+i L_{\mu} U^{-1}-i U^{-1} R_{\mu}$. Since we are interested in effective action for QCD we set $L_{\mu}=R_{\mu}=G_{\mu}$ in eq. (4.4), to obtain

$$
\begin{gathered}
S_{\mathrm{eff}}=\frac{\Lambda^{2}}{2 \pi^{2}} \int \operatorname{Tr}\left(D_{\mu} U D^{\mu} U^{-1}\right)-\frac{1}{240 \pi^{2}} \int \epsilon^{\mu \nu \lambda \sigma \rho} \operatorname{Tr}\left(J_{\mu} J_{\nu} J_{\lambda} J_{\sigma} J_{\rho}\right) \\
+\frac{i}{48 \pi^{2}} \int \epsilon^{\mu \nu \lambda \sigma} \operatorname{Tr}\left\{i G_{\mu \nu}\left[G_{\lambda}, \bar{J}_{\sigma}\right]-\left(G_{\mu} G_{\nu}-\frac{3}{2} G_{\mu} \bar{J}_{\nu}+\bar{J}_{\mu} \bar{J}_{\nu}\right) G_{\lambda} \bar{J}_{\sigma}\right\} \\
-\frac{i}{48 \pi^{2}} \int \epsilon^{\mu \nu \lambda \sigma} \operatorname{Tr}\left[i\left(U^{-1} G_{\mu \nu} U+G_{\mu \nu}\right)\left\{G_{\lambda}, G_{\sigma}^{U}\right\}+\left(G_{\mu} G_{\nu}-\frac{1}{2} G_{\mu} G_{\nu}^{U}+G_{\mu}^{U} G_{\nu}^{U}\right) G_{\lambda} G_{\sigma}^{U}\right] \\
+\frac{1}{192 \pi^{2}} \int \operatorname{Tr}\left[-2\left(D_{\mu} U^{-1} D^{\mu} U\right)^{2}+\left(D_{\mu} U^{-1} D_{\nu} U\right)^{2}+2\left(D_{\mu} D^{\mu} U^{-1}\right) D_{\nu} D^{\nu} U\right. \\
\left.+4\left(G_{\mu \nu} D^{\mu} U^{-1} D^{\nu} U+G_{\mu \nu} D^{\mu} U D^{\nu} U^{-1}\right)+U^{-1} G_{\mu \nu} U G^{\mu \nu}-G_{\mu \nu} G^{\mu \nu}\right]
\end{gathered}
$$




\section{Bosonization Schemes and Solutions}

\subsection{DIFFERENT SCHEMES}

In this section we introduce several schemes of the effective action. In the previous section we derived the effective low-energy action for a general coset $G / H$ with $H$ a subgroup of $G$. The chiral phase takes values in $G$, while the gauge field takes values in $H$. In QCD, $H$ is $S U\left(N_{c}\right)$. Depending on the physics one is interested in, there are various choices for $G$. We list here those which we consider the most important.

1. The " $U$-scheme". In this case we choose $U \in U\left(N_{c} \times N_{f}\right)$.

2. The "product scheme". The group $G$ is $S U\left(N_{c}\right) \times S U\left(N_{f}\right) \times U(1)$. One can parameterize $U$ by $U=h \cdot g$, where $h \in S U\left(N_{c}\right)$ and $g \in U\left(N_{f}\right)$.

3. The effective $U(1)$ action. It is an effective action for the $\eta^{\prime}$. It is related to $\operatorname{det} U^{[23]}$.

\subsection{Classical solutions in the $U$-SCheme}

The classical soliton solutions are embedded in an $S U(2)$ subgroup of $U\left(N_{c} N_{f}\right)$. They have the form

$$
U_{c}=e^{i f(r) \vec{\tau} \cdot \hat{r}}
$$

where $\vec{\tau}$ are the generators of this $S U(2)$ subgroup and $f(r)$ is a radial shape function with boundary conditions $f(0)=\pi, f(\infty)=0$. There are three choices for $\vec{\tau}$.

1. $\vec{\tau}=\vec{\sigma}_{c} \times E_{f}$, where $E_{f}$ is a matrix in flavor space which satisfies the relation $E_{f}^{2}=E_{f}$. This is a colored soliton. This kind of soliton has been discussed in detail by Kaplan. ${ }^{[9]}$ Its baryon number is $\operatorname{Tr}_{f}\left(E_{f}\right)$.

2. $\vec{\tau}=E_{c} \times \vec{\sigma}_{f}$. If $E_{c}$ is the identity, this is a color-singlet flavored soliton. This is the familiar Skyrme type soliton ${ }^{[6,7]}$, widely discussed in the literature. Its baryon number is $\operatorname{Tr}_{c}\left(E_{c}\right)$. 
3. A mixed colored flavored soliton. From the general discussion in Ref. 9 and from the calculations in 2 dimensions ${ }^{[11]}$ we expect that this new type of soliton corresponds to a constituent quark.

The solitons of the theory can be taken in color space using the following ansatz,

$$
U_{c}^{i}=e^{i f(r)\left(\vec{\tau}_{c} \cdot \vec{r}\right) E_{f}^{i}}
$$

where $\vec{\tau}_{c}$ is in an $S U(2)$ subgroup of $S U\left(N_{c}\right)$ and $E_{f}^{i}$ is a matrix in flavor space with 1 in the $(i, i)$ entry and zeroes elsewhere. In the normalization convention in which a nucleon has baryon number $N_{c}$, the baryon number of this soliton is 1 and its flavor quantum number is $i$, i.e. it has the quantum numbers of a quark.

It is important to keep in mind, however, that the classical solution with the quantum numbers of a constituent quark has a finite energy. This is in clear contrast with the expectation that there are no finite energy colored states. This is a clear deficiency of the present treatment, and it can be traced back to the fact that in the anomaly integration approach, confinement has to be put in by hand, as it comes from the terms which we do not treat.

One may also consider solutions which carry baryon number different from 1. In view of the results in 2 dimensions ${ }^{[11]}$ we expect to find color-singlet solutions constructed out of soliton-anti-soliton pair and solutions which are constructed from $N_{c}$ solitons. It is natural to interpret such solutions as mesons and baryons and their constituent solitons as constituent quarks.

\subsection{CONSERVED CURRENTS}

The right and left currents are given by

$$
\begin{aligned}
& \frac{\delta S_{\mathrm{eff}}}{\delta R_{\mu}}=\frac{\Lambda^{2}}{\pi^{2}} \bar{J}^{\mu}-\frac{i}{48 \pi^{2}} \epsilon^{\mu \nu \lambda \sigma} \bar{J}_{\nu} \bar{J}_{\lambda} \bar{J}_{\sigma}-\frac{1}{48 \pi^{2}}\left(\left\{\bar{J}_{\nu} \bar{J}^{\nu}, \bar{J}^{\mu}\right\}-\bar{J}_{\nu} \bar{J}^{\mu} \bar{J}^{\nu}\right) \\
& \frac{\delta S_{\mathrm{eff}}}{\delta L_{\mu}}=\frac{\Lambda^{2}}{\pi^{2}} J^{\mu}+\frac{i}{48 \pi^{2}} \epsilon^{\mu \nu \lambda \sigma} J_{\nu} J_{\lambda} J_{\sigma}-\frac{1}{48 \pi^{2}}\left(\left\{J_{\nu} J^{\nu}, J^{\mu}\right\}-J_{\nu} J^{\mu} J^{\nu}\right)
\end{aligned}
$$


and the baryon number current by the trace of their sum,

$$
J_{\mu}^{B}=\frac{i}{24 \pi^{2}} \epsilon^{\mu \nu \lambda \sigma} \operatorname{Tr}\left(J_{\nu} J_{\lambda} J_{\sigma}\right)
$$

Eqs. (5.2) and (5.3) can be conveniently written in a compact form

$$
\begin{aligned}
& \frac{\delta S_{\mathrm{eff}}}{\delta R_{\mu}}=\operatorname{Tr}\left[\frac{1-\gamma_{5}}{2} \gamma^{\mu}, \bar{J}\left(\frac{\Lambda^{2}}{2 \pi^{2}}-\frac{1}{96 \pi^{2}} \bar{\nearrow}, \bar{J}\right)\right] \\
& \frac{\delta S_{\text {eff }}}{\delta L_{\mu}}=\operatorname{Tr}\left[\frac{1+\gamma_{5}}{2} \gamma^{\mu}, \not\left(\frac{\Lambda^{2}}{2 \pi^{2}}-\frac{1}{96 \pi^{2}}, \not, J\right)\right]
\end{aligned}
$$

\subsection{The Polyakov-Wiegmann formula ${ }^{[24]}$}

We want to find the effective action for 2 chiral phases. The basic identity follows from the definition of the effective action $S_{\text {eff }}(2.11)$.

$$
S_{\text {eff }}\left[L, R, U_{1} U_{2}\right]=S_{\text {eff }}\left[L, R^{U_{1}}, U_{2}\right]+S_{\text {eff }}\left[L, R, U_{1}\right]
$$

For zero sources we have

$$
S_{\text {eff }}\left[0,0, U_{1} U_{2}\right]=S_{\text {eff }}\left[0,0, U_{1}\right]+S_{\text {eff }}\left[0,0, U_{2}\right]+S_{1}\left[U_{1}, U_{2}\right]
$$

which defines $S_{1}\left[U_{1}, U_{2}\right]$ as

$$
S_{1}\left[U_{1}, U_{2}\right]=S_{\mathrm{eff}}\left[0,-J_{1}^{\mu}, U_{2}\right]-S_{\mathrm{eff}}\left[0,0, U_{2}\right]
$$

For 2 dimensions we have ${ }^{[24]}$

$$
S_{\text {eff }}[0,0, U]=\frac{1}{8 \pi} \int \operatorname{Tr}\left(J_{\mu} J^{\mu}\right)-\frac{i}{12 \pi} \int \epsilon^{\mu \nu \sigma} \operatorname{Tr}\left(J_{\mu} J_{\nu} J_{\sigma}\right)
$$


and

$$
S_{1}=\left[U_{1}, U_{2}\right]=-\frac{1}{4 \pi} \int\left(g_{\mu \nu}+\epsilon_{\mu \nu}\right) \operatorname{Tr}\left(J_{2}^{\mu} \bar{J}_{1}^{\nu}\right)
$$

For 4 dimensions we have

$$
\begin{gathered}
S_{\text {eff }}[0,0, U]=\frac{\Lambda^{2}}{2 \pi^{2}} \int \operatorname{Tr}\left(J_{\mu} J^{\mu}\right)-\frac{1}{240 \pi^{2}} \int \epsilon^{\mu \nu \lambda \sigma \rho} \operatorname{Tr}\left(J_{\mu} J_{\nu} J_{\lambda} J_{\sigma} J_{\rho}\right) \\
+\frac{1}{192 \pi^{2}} \int \operatorname{Tr}\left[-2\left(J_{\mu} J^{\mu}\right)^{2}+\left(J_{\mu} J_{\nu}\right)^{2}+2\left(\partial_{\mu} \partial^{\mu} U^{-1}\right) \partial_{\nu} \partial^{\nu} U\right] \\
S_{1}\left[U_{1}, U_{2}\right]=-\frac{\Lambda^{2}}{\pi^{2}} \int \operatorname{Tr} \bar{J}_{2}^{\mu} J_{1}^{\mu}+\frac{1}{48 \pi^{2}} \int \epsilon_{\mu \nu \lambda \sigma} \operatorname{Tr}\left(J_{1}^{\mu} J_{1}^{\nu} J_{1}^{\lambda} \bar{J}_{2}^{\sigma}\right. \\
\left.+\frac{3}{2} J_{1}^{\mu} \bar{J}_{2}^{\nu} J_{1}^{\lambda} \bar{J}_{2}^{\sigma}-\bar{J}_{2}^{\mu} \bar{J}_{2}^{\nu} \bar{J}_{2}^{\lambda} J_{1}^{\sigma}\right)
\end{gathered}
$$

\section{Summary and outlook}

We have derived an effective low energy action for QCD in 4 dimensions. We have shown that the solitons in the theory have quantum numbers of quarks. We interpret these solitons as constituent quarks. An interesting plan might be to calculate the equations of motions numerically and compare the results to experimental values. Other quantities such as the $g_{A}$ of the constituent quarks might be calculated. In this case $g_{A}$ has a classical contribution too, in analogy with the Skyrme model. Multi-soliton solutions can also be considered as baryons and mesons constructed out of quark solitons.

Acknowledgements: The authors would like to thank John Ellis for useful discussions. Y.F. would like to acknowledge interesting discussions with W. Bardeen. A.H. Would like to thank Adam Schwimmer, Jacob Sonnenschein and Yigal Shamir for useful discussions.

The research of Y.F. and A.H. was supported in part by the Basic Research Foundation administered by the Israel Academy of Sciences and Humanities. The 
research of M.K. was supported in part by the Einstein Center at the Weizmann Institute, by the Basic Research Foundation administered by the Israel Academy of Sciences and Humanities and by grant No. 90-00342 from the United States-Israel Binational Science Foundation(BSF), Jerusalem, Israel.

Note Added: Upon the completion of this work we have received a draft of the paper by Damgaard and Sollacher ${ }^{[25]}$ in which the effective action in four dimensions is derived by closely related techniques. We thank the authors for communicating their results to us prior to publication.

\section{REFERENCES}

1. The EMC Collaboration, J. Ashman et al., Phys. Lett. B206(1988)364; Nucl. Phys. B328(1989)1.

2. The SMC Collaboration, B. Adeva et al., Phys. Lett. B302(1993)533.

3. The E142 Collaboration, P.L. Anthony et al., Phys. Rev. Lett. 71(1993)959.

4. For recent theoretical analysis of the data see J. Ellis and M. Karliner, Phys. Lett. B313(1993)131 and J. Ellis and M. Karliner, Spin Structure Functions; Plenary talk at PANIC '93, CERN-TH.7022/93, hep-ph/9310272, and references therein.

5. A. Manohar and H. Georgi, Nucl. Phys. B234(1984)189.

6. T. H. R. Skyrme, Proc. Roy. Soc. London B260(1961)127.

7. E. Witten, Nucl. Phys. B223(1983)422 and 433; G. Adkins, C. Nappi, and E. Witten, Nucl. Phys. B228(1983)552; $S U(3)_{f}$ extension in E. Guadagnini, Nucl. Phys. B236(1984)35; P. O. Mazur, M. A. Nowak, and M. Praszałowicz, Phys. Lett. B147(1984)137.

8. E. Witten in Lewes Workshop Proc.; A. Chodos et al., Eds; Singapore, World Scientific, 1984. 
9. D. B. Kaplan, Phys. Lett.B235(1990)163; Nucl. Phys.B351,(1991)137.

10. G. Gomelski, M. Karliner and S. B. Selipsky, Static properties of quark solitons, BUHEP-92-17/TAUP 2034-93, hep-ph/9304217 (1993).

11. J. Ellis, Y. Frishman, A. Hanany and M. Karliner, Nucl. Phys. B382(1992)189.

12. A. A. Andrianov and L. Bonora, Nucl. Phys. B233(1984)232.

13. J. Balog, Phys. Lett. B149(1984)197.

14. J. L. Manes, Nucl. Phys. B250(1985)369.

15. W. A. Bardeen and B. Zumino, Nucl. Phys. B244(1984)421.

16. K. Fujikawa Phys. Rev. D21(1980)2848; erratum ibid., D22(1980)1499.

17. J. Gasser and H. Leutwyler Nucl. Phys. B250(1985)465.

18. D. Espriu, E. de Rafael and J. Taron, Nucl. Phys. B345(1990)22.

19. E. Witten, Comm. Math. Phys 92(1984)455.

20. Y. Frishman Quark trapping in a model field theory. Mexico City 1973. Berlin, Heidelberg, New York: Springer 1975

21. W. A. Bardeen, Phys. Rev. 184(1969)1848.

22. L. Alvarez-Gaume and P. Ginsparg, Ann. Phys. 161(1985)423.

23. G. Veneziano, $C P$ and $U(1)$ problems, and their relationship in $Q C D$; in La Plagne 1983 Proceedings, Beyond The Standard Model, pp. 355-367.

24. A. Polyakov and P. B. Wiegmann, Phys. Lett. B131(1983)121.

25. P.H. Damgaard and R. Sollacher, CERN-TH-7073/93, Nov. 1993, submitted to Phys. Lett. 